\section{Fluxo de carga durante a fase fluente de recomposição do sistema elétrico: estudo de caso do subsistema sudoeste da região Nordeste do Brasil}

\section{Charge flow during fluent phase of rebuilding electrical system: a case study of southwestern subsystem of northeastern Brazil}

\section{Flujo de carga durante la fase fluente de recomposición del sistema eléctrico: estudio de caso del subsistema sudoeste de la región Nordeste de Brasil}

\section{Resumo}

A recomposição do sistema elétrico após uma perturbação acontece em duas fases: fase fluente e fase coordenada. A fase fluente é a mais sensível, pois é quando o sistema opera de forma ilhada, partindo de uma usina de autorrestabelecimento. O Sistema Interligado Nacional (SIN) é organizado de modo que as regiões que o compõem possuam essas usinas, responsáveis por iniciar a reenergização do sistema após uma falha. Uma dessas usinas é a usina de Sobradinho, que integra o subsistema sudoeste da região Nordeste. Assim, a análise do fluxo de carga durante o processo de recomposição deve ser levada em consideração para que a retomada ocorra de forma segura e eficaz. O presente artigo apresenta um estudo do fluxo de cargas durante a fase fluente do processo de reenergização do subsistema sudoeste da região Nordeste, a partir da análise computacional. Para tanto, fez-se o modelamento do sistema no programa PowerWorld seguindo as instruções normativas operacionais do Operador Nacional do Sistema, o ONS. Portanto, os resultados obtidos encontram-se em conformidade com o esperado nas instruções normativas, além de indicar quais recursos de regulação do sistema podem ser otimizados, visando a melhoria no processo de recomposição do sistema elétrico.

Palavras-chave: Sistema Elétrico, Recomposição, Fase Fluente, Fluxo de Carga, PowerWorld.

\begin{abstract}
The rebuilding of the electrical system after a disturbance occurs in two phases: fluent phase and coordinated phase. The fluent one is the most sensitive because it is the place the system operates in an isolated way, starting from a self-healing plant. The National Interconnected System (SIN) is organized in such a way that the regions that comprise it have these plants, which are responsible for initiating the re-energization of the system after a failure. Sobradinho is one of these plants, which is part of the Southwest subsystem of the Northeast region. Thus, the analysis of the charge flow during the rebuilding process must be considered so that the resumption occurs safely and effectively. This article presents a study charges flow during the re-energization fluent phase process of the southwestern subsystem in the Northeast region, based on computational analysis. To this end, the system was modelled using PowerWorld software following the operational normative instructions of the National System Operator, ONS. Therefore, the results obtained follow the expected in normative instructions in addition to indicating that the system's regulatory resources can be optimized, aiming the improvement of the rebuilding electrical system process.
\end{abstract}

Keywords: Electrical System. Rebuilding. Fluent Phase. Charge Flow. PowerWorld. 


\begin{abstract}
Resumen
La recomposición del sistema eléctrico luego después de una perturbación ocurre en dos fases: fase fluente y fase condenada. La fase fluente es la más sensible, pues el sistema opera de forma aislada, saliendo de una usina de auto restablecimiento. El Sistema Interconectado Nacional (SIN) es organizado de modo que las regiones que lo componen posean esas usinas, responsables por iniciar la reactivar la energía del sistema tras un fallo. Una de estas usinas es la de Sobradinho, que compone el subsistema sudoeste de la región Nordeste. Así, el análisis del flujo de carga durante el proceso de recomposición debe ser levada en consideración para que la retomada ocurra de forma segura y eficaz. Es artículo presenta un estudio del flujo de cargas durante la fase fluente del proceso de reactivación de energía del subsistema sudoeste de la región Nordeste, a partir del análisis computacional. Para tanto, se hizo un modelado del sistema en el programa PowerWorld siguiendo las instrucciones normativas operacionales del Operador Nacional del Sistema, el ONS. Por lo tanto, los resultados obtenidos se encuentran en conformidad con el esperado en las instrucciones normativas, además de indicar cuales recursos de regulación del sistema pueden ser optimizados, objetivando la mejora en el proceso de recomposición del sistema eléctrico.
\end{abstract}

Palabras-clave: Sistema Eléctrico. Recomposición. Fase Fluente. Flujo de Carga. PowerWorld.

\section{Introdução}

O principal objetivo de um Sistema Elétrico de Potência (SEP) é gerar, transmitir e distribuir energia elétrica, em conformidade com determinados padrões de confiabilidade, disponibilidade, custo e com o menor impacto ambiental possível (STEVENSON, 1986).

A vida em sociedade da forma como conhecemos só é possível graças ao suprimento constante de energia elétrica. Por isso, para garantir a continuidade do fornecimento de energia, os sistemas elétricos operam interligados. Assim, de acordo com Kindermann (2010) o comportamento do sistema elétrico deve ser feito de maneira sistemática e constante.

Por conta da atual configuração da sociedade, uma falha no sistema elétrico pode gerar prejuízos que vão desde perdas financeiras, até mesmo perdas de vidas de pacientes que porventura estejam ligados a aparelhos nos hospitais. Por este motivo, os responsáveis pela operação do sistema devem trabalhar para que em caso de falha, a recomposição seja feita de maneira rápida, segura e eficaz. Para tanto se faz necessário compreender o fluxo de carga no sistema.

O objetivo deste estudo foi analisar o fluxo de cargas durante a fase fluente de recomposição do subsistema sudoeste da região Nordeste do Brasil, a partir do modelamento computacional do sistema. Para tanto foi utilizado o programa PowerWorld Simulator e foram seguidas as orientações da instrução IO-RR.NE.SBSO do Operador Nacional do Sistema (ONS) que trata do tema.

\section{Operação do sistema elétrico}

O Sistema Interligado Nacional (SIN) é formado por uma série de equipamentos como usinas, linhas de transmissão, transformadores dentre outros, responsáveis por conectar geração, transmissão, distribuição e consumo em todo território nacional. A confiabilidade do sistema é garantida pela operação segura destes equipamentos, atendendo às instruções normativas de operação, nos mais diversos níveis de tensão, interligando subestações e regiões, levando energia elétrica gerada pelas mais variadas fontes (hidráulica, térmica, solar, eólica, dentre outras) ao consumidor final.

No sistema elétrico brasileiro predominam-se as usinas hidrelétricas com múltiplos proprietários. Conforme o ONS (ONS, 2017a) o SIN é constituído de quatro subsistemas: Sul, Sudeste/Centro-Oeste, Nordeste e Norte. Na Figura 1 é possível observar o mapa do sistema elétrico de transmissão brasileiro atualizado e com projeção até 2024. 
Figura 1: Mapa do sistema de transmissão brasileiro

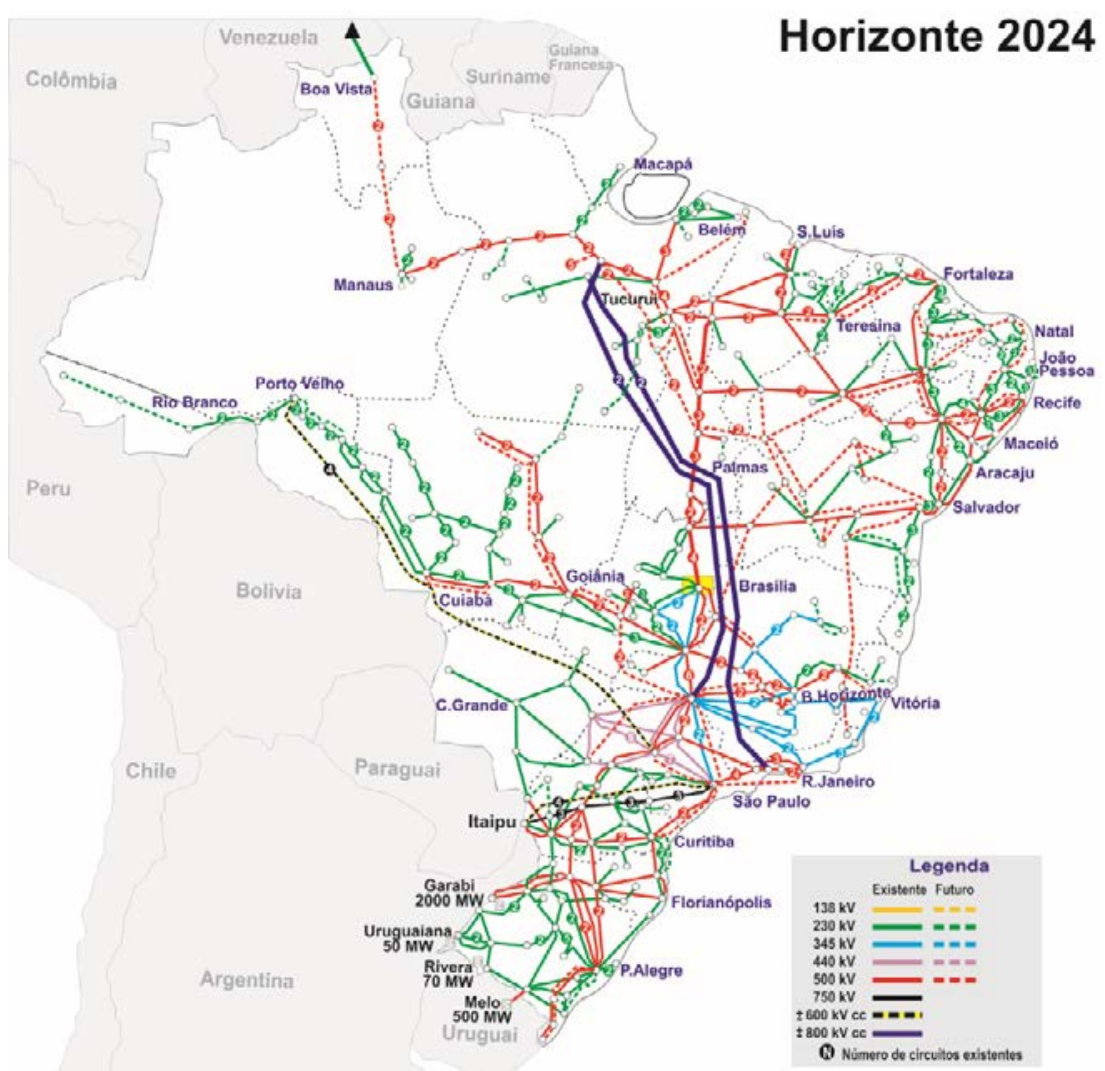

Fonte: ONS, 2020.

Uma característica importante, que deve ser levada em consideração ao se estudar um sistema elétrico, é como a carga no sistema evolui. A Figura 2 mostra a projeção de carga anual para o sistema brasileiro. $\mathrm{O}$ estudo do fluxo de cargas revela-se importante por dois motivos principais: contribuir para melhorar as condições atuais do sistema e acompanhar a demanda de energia elétrica, para prever necessidade de ampliação ou recapacitação de equipamentos. O Boletim Técnico ONS/CEE/EPE - "Previsões de Carga para o Planejamento Anual da Operação Energética 2017-2021 - 1ª Revisão Quadrimestral" traz as previsões de cargas que foram adotadas neste trabalho.

Figura 2: Projeção anual de carga (MWmed) brasileira

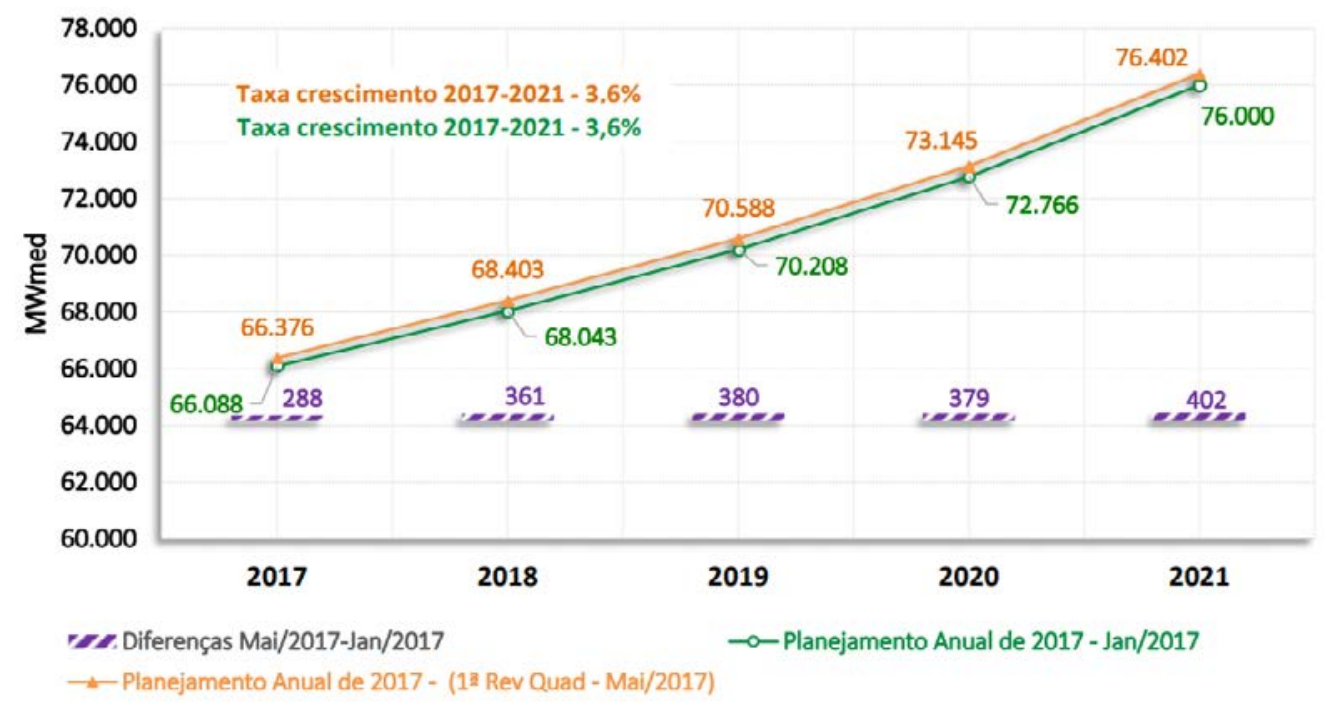

Fonte: ONS, 2017b. 


\subsection{Perturbações no sistema elétrico}

O ONS define perturbação no item 302 do submódulo 20.1 - glossário de termos técnicos dos procedimentos de rede — da seguinte maneira: "Ocorrência no SIN caracterizada pelo desligamento forçado de um ou mais de seus componentes, que acarreta quaisquer das seguintes consequências: corte de carga, desligamento de outros componentes do sistema, danos em equipamentos ou violação de limites operativos" (ONS, 2017c, p.33).

As perturbações no sistema elétrico podem ter causas diversas e suas consequências são variadas: desde uma simples falta de energia, com rápido restabelecimento; até o desligamento geral do sistema. Uma perturbação pode ser ainda resultado de um ato de retirada indevida de serviço de um equipamento ou LT (linha de transmissão), que esteja operando em condições anormais, resultante de falhas, por desligamento automático ou de emergência. Independente da causa do desligamento, as equipes de manutenção e operação devem atuar de forma rápida e eficaz para o restabelecimento das condições normais de fornecimento de energia, com o objetivo de minimizar os prejuízos à sociedade (sejam financeiros, operacionais, ou mesmo com perdas de vidas que dependem de aparelhos em hospitais).

\subsection{Operação normal e recomposição após perturbação}

A operação normal do sistema é feita com base no monitoramento e acompanhamento dos equipamentos pelo ONS e pelos agentes responsáveis (ONS, 2017d). São feitas, então, ações de coordenação, medição, supervisão e controle dos níveis de tensão dos barramentos e dos carregamentos. Devido ao dinamismo da carga, durante o dia, existem períodos em que as variações são mais acentuadas. Portanto, torna-se necessário manter o equilíbrio entre o que a sociedade está consumindo de energia e o que está sendo produzido pela geração.

A regulação de tensão é fundamental para manter o equilíbrio do sistema. Neste aspecto o subsistema sudoeste, foco deste estudo, apresenta alguns pontos relevantes. Primeiro, na usina de Sobradinho os geradores (por conta de suas características construtivas e operacionais) podem atuar como compensadores síncronos. Em segundo lugar, as usinas eólicas também operam como reguladores de tensão, pois além do fornecimento de potência ativa, fornecem e absorvem o reativo no sistema. Por fim, as subestações do sistema também atuam na regulação da tensão, pois possuem mecanismos que controlam o fornecimento e absorção do reativo do sistema. (MATOS, 2017).

\subsection{Usinas de autorrestabelecimento}

Uma usina de autorrestabelecimento é aquela em que se inicia o processo de recomposição do sistema após um desligamento geral. Conforme definição do ONS, "são usinas que possuem uma ou mais unidades com capacidade de autorrestabelecimento, estando todas as suas unidades geradoras na condição de repouso - desligadas elétrica e mecanicamente - independentemente de qualquer alimentação externa para suprir os seus serviços auxiliares" (ONS, 2017e, p.4). Esses tipos de usinas são capazes de, com seus próprios recursos de serviços auxiliares, dar início à partida nas unidades e sincronizar o número mínimo de unidades definido nas instruções de operação de recomposição do ONS.

A recomposição da Rede de Operação após perturbação é uma atividade de tempo real que visa restabelecer com rapidez e segurança a condição normal de operação do Sistema Interligado Nacional - SIN, com o atendimento pleno da carga. O processo de recomposição do sistema é composto de duas fases, a fase fluente e a fase coordenada (ONS, 2017d, p.3).

O estudo aqui realizado analisa o fluxo de carga na fase fluente do processo de recomposição. Na fase fluente o sistema opera de forma ilhada, partindo de uma usina de autorrestabelecimento integral, chegando a um determinado número de instalações e cargas.

\subsection{O Subsistema elétrico sudoeste da região Nordeste}

O Quadro 1 traz as instalações da área de Sobradinho-Sudoeste da Região Nordeste, conforme estrutura definida pelo ONS (ONS, 2017f). Na primeira linha veem-se as instalações que fazem parte da fase fluente de recomposição, enquanto na segunda linha veem-se as instalações da fase coordenada. 
Quadro 1: Instalações da área Sobradinho-Sudoeste da região Nordeste.

\begin{tabular}{|c|c|c|c|c|c|c|}
\hline $\begin{array}{c}\text { UHE/SE } \\
\text { Sobradinho }\end{array}$ & $\begin{array}{c}\text { SE Juazeiro } \\
\text { da Bahia II }\end{array}$ & SE Jaguarari & $\begin{array}{c}\text { SE Senhor do } \\
\text { Bonfim II }\end{array}$ & $\begin{array}{c}\text { SE Campo } \\
\text { Formoso }\end{array}$ & SE Irecê & $\begin{array}{c}\text { SE Brotas de } \\
\text { Macaúbas }\end{array}$ \\
\hline SE Barreiras & $\begin{array}{c}\text { SE Bom Jesus } \\
\text { da Lapa }\end{array}$ & $\begin{array}{c}\text { SE Bom Jesus } \\
\text { da Lapa II }\end{array}$ & $\begin{array}{c}\text { Tabocas do } \\
\text { Brejo Velho }\end{array}$ & $\begin{array}{c}\text { SE Pedra } \\
\text { Branca }\end{array}$ & SE Igaporã II & $\begin{array}{c}\text { SE Rio Grande } \\
\text { II }\end{array}$ \\
\hline
\end{tabular}

Fonte: ONS (2017f).

O esquema elétrico do subsistema analisado está representado na Figura 3. Inicialmente tem-se a usina de Sobradinho, em seguida a subestação de $500 \mathrm{kV}$ de Sobradinho no qual existem 3 autotransformadores de $500 \mathrm{kV}$ para $230 \mathrm{kV}$, conectando o eixo à subestação Brotas de Macaúbas (eixo identificado na cor verde). Na Figura 3 é possível ver também os pontos de conexão com as cargas de 69 kV e 138 kV, identificadas nas cores azul e laranja respectivamente. Ressalta-se que somente a fase fluente é alvo deste estudo.

Figura 3: Subsistema sudoeste da região Nordeste fase fluente

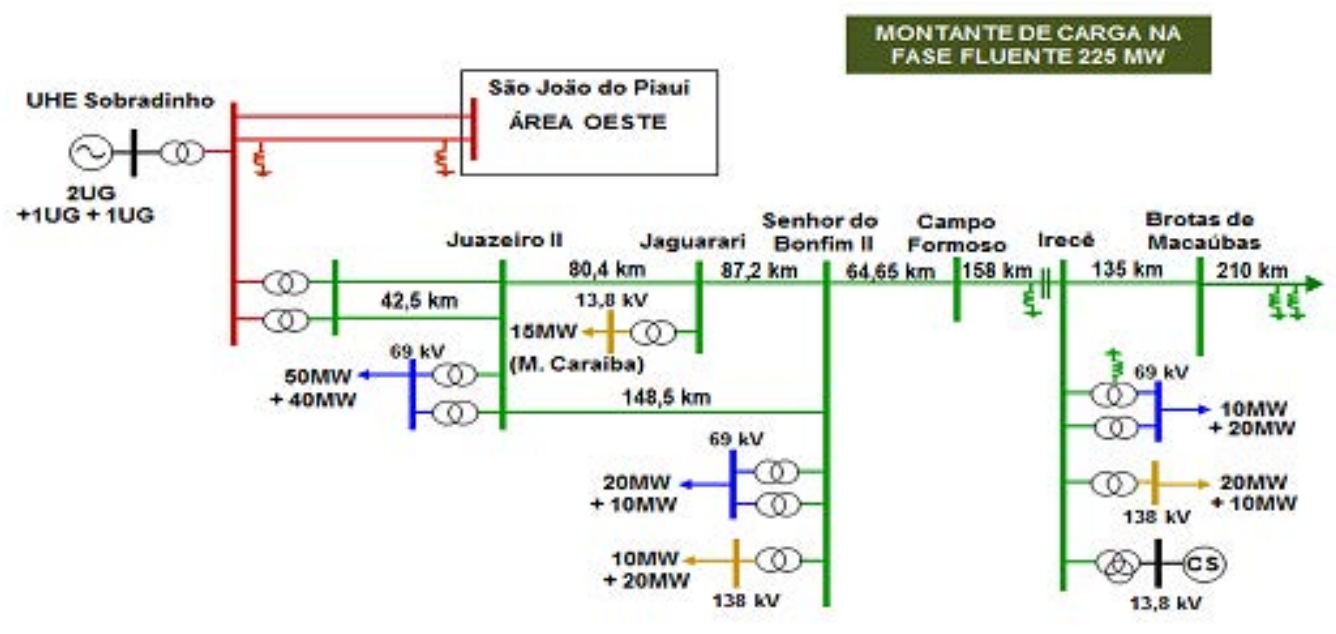

Fonte: ONS, $2017 f$ (adaptada).

O Quadro 2 traz a descrição e a composição das instalações do subsistema sudoeste, fase fluente.

Quadro 2: Descrição e composição das instalações do subsistema sudoeste fase fluente

\begin{tabular}{|c|c|c|c|}
\hline Instalação & Sigla & Descrição & Composição \\
\hline Usina de Sobradinho & USB & $\begin{array}{l}\text { Usina hidrelétrica localizada no rio São Francisco. } \\
\text { Cada transformador elevador é conectado a um } \\
\text { par de geradores. }\end{array}$ & $\begin{array}{c}6 \text { Geradores de } 175,05 \mathrm{MW}, 13,8 \mathrm{kV} \\
\mathrm{fp} 0,9 \mathrm{e} \\
3 \text { transformadores } 13,8 \mathrm{kV} / 500 \mathrm{kV}\end{array}$ \\
\hline Subestação Sobradinho & SOB & $\begin{array}{l}\text { Subestação de } 500 \mathrm{KV} \text {. Responsável por suprir } \\
\text { todo o eixo } 230 \mathrm{kV} \text { do subsistema. Capacidade } \\
\text { de operação de } 900 \text { MVA. }\end{array}$ & 3 Autotransformadores $500 \mathrm{kV} / 230 \mathrm{kV}$ \\
\hline Subestação Juazeiro II & JZD & $\begin{array}{l}\text { Subestação abaixadora. Capacidade de operação } \\
\text { de } 400 \text { MVA. Alimenta as cargas das distribuidoras } \\
\text { regionais COELBA E CELPE. }\end{array}$ & 4 Transformadores 230 kV/ 69 kV \\
\hline Subestação Jaguarari & JGR & $\begin{array}{l}\text { Alimenta um consumidor industrial. Capacidade } \\
\text { de operação de } 120 \text { MVA. }\end{array}$ & 2 Transformadores $230 \mathrm{kV} / 13,8 \mathrm{kV}$ \\
\hline Subestação Senhor do Bonfim II & SNB & $\begin{array}{l}\text { Subestação abaixadora. Pode transformar de } 230 \\
\text { kV para } 69 \mathrm{kV} \text { ou para } 138 \mathrm{kV} \text {. Capacidade de } \\
\text { operação de } 400 \mathrm{MVA}\end{array}$ & $\begin{array}{l}2 \text { Transformadores } 230 \mathrm{kV} / 138 \mathrm{kV} \text { e } \\
2 \text { transformadores } 230 \mathrm{kV} / 69 \mathrm{kV}\end{array}$ \\
\hline Subestação Campo Formoso & CFO & $\begin{array}{l}\text { Subestação } 230 \text { kV. } \\
\text { Recebe energia eólica produzida. }\end{array}$ & 2 Transformadores $230 \mathrm{kV} / 34.5 \mathrm{kV}$ \\
\hline Subestação Irecê & IRE & $\begin{array}{l}\text { Subestação abaixadora da qual saem quatro linhas } \\
\text { em } 69 \mathrm{kV} \text { e uma linha em } 138 \mathrm{kV} \text {. Capacidade } \\
\text { total de } 227 \mathrm{MVA} \text {. }\end{array}$ & $\begin{array}{c}3 \text { Transformadores } 230 \mathrm{kV} / 69 \mathrm{kV} \mathrm{e} \\
2 \text { autotransformadores } 230 \mathrm{kV} / 138 \mathrm{kV}\end{array}$ \\
\hline Subestação Brotas de Macaúbas & BMC & $\begin{array}{l}\text { Subestação em } 230 \text { kV. Interliga as subestações } \\
\text { Irecê e Bom Jesus da Lapa. }\end{array}$ & $\begin{array}{l}1 \text { Transformador } \\
230 \mathrm{kV} / 34.5 \mathrm{kV}\end{array}$ \\
\hline
\end{tabular}

Fonte: Elaboração própria (2017). 
Além das instalações mencionadas no Quadro 2, compõem também o subsistema sudoeste as linhas de transmissão. Os dados dos limites operacionais e dos comprimentos das linhas foram retirados de documento do ONS que trata do cadastro de limites operacionais das linhas de transmissão e transformadores da área $230 \mathrm{kV}$ sudoeste da região Nordeste (ONS, 2017g).

Quadro 3: Linhas de transmissão do subsistema sudoeste fase fluente

\begin{tabular}{lccc}
\hline \multicolumn{1}{c}{ Linha } & Comprimento & Tensão & Capacidade de corrente \\
\hline Interligação SOB-JZD & $42,5 \mathrm{~km}$ (duas linhas) & $230 \mathrm{kV}$ (cada) & $879 \mathrm{~A}$ (cada) \\
Interligação JZD-JGR & $80,4 \mathrm{~km}$ & $230 \mathrm{kV}$ & $631 \mathrm{~A}$ \\
Interligação JGR-SNB & $87,2 \mathrm{~km}$ & $230 \mathrm{kV}$ & $631 \mathrm{~A}$ \\
Interligação JZD-SNB & $148,5 \mathrm{~km}$ & $230 \mathrm{kV}$ & $631 \mathrm{~A}$ \\
Interligação SNB-CFO-IRE & $64,65 \mathrm{~km}$ (SNB-CFO) & $230 \mathrm{kV}$ (cada) & $631 \mathrm{~A}$ (cada) \\
Interligação IRE-BMC & $158 \mathrm{~km}$ (CFO-IRE) & & $600 \mathrm{~A}$ \\
\hline
\end{tabular}

Fonte: Elaboração própria (2017).

Após o apagão de 2011, o ONS elaborou as instruções de operação relacionadas a seguir. Tais normativas tem objetivo de coordenar a recomposição do sistema permitindo que a energia chegue aos centros urbanos de maneira mais rápida e eficaz. São elas:

IO-RR.NE.UXG - recomposição da área Xingó;

IO-RR.NE.ULG - recomposição da área Luiz Gonzaga;

IO-RR.NE.PAQ - recomposição da área Paulo Afonso IV;

IO-RR.NE.UITP - recomposição da área Itapebi;

IO-RR.NE.SBOE - recomposição da área Sobradinho - Oeste;

IO-RR.NE.SBSO - recomposição da área Sobradinho - Sudoeste.

A usina de Sobradinho (USB) é a principal fonte de energia do caso estudado. A USB é a usina de autorrestabelecimento do subsistema sudoeste da região Nordeste.

A USB é uma usina de autorrestabelecimento integral, capaz de energizar os elementos da rede adjacente, como transformadores, autotransformadores e linhas de transmissão, sem ter que considerar nenhum evento que esteja fora do seu controle, absorver variações bruscas de carga, bem como, controlar as tensões e frequência dentro das faixas definidas para situação de emergência (MATOS, 2017, p.25).

O diagrama unifilar da USB é mostrado na Figura 4. 
Figura 4: Diagrama unifilar da Usina de Sobradinho

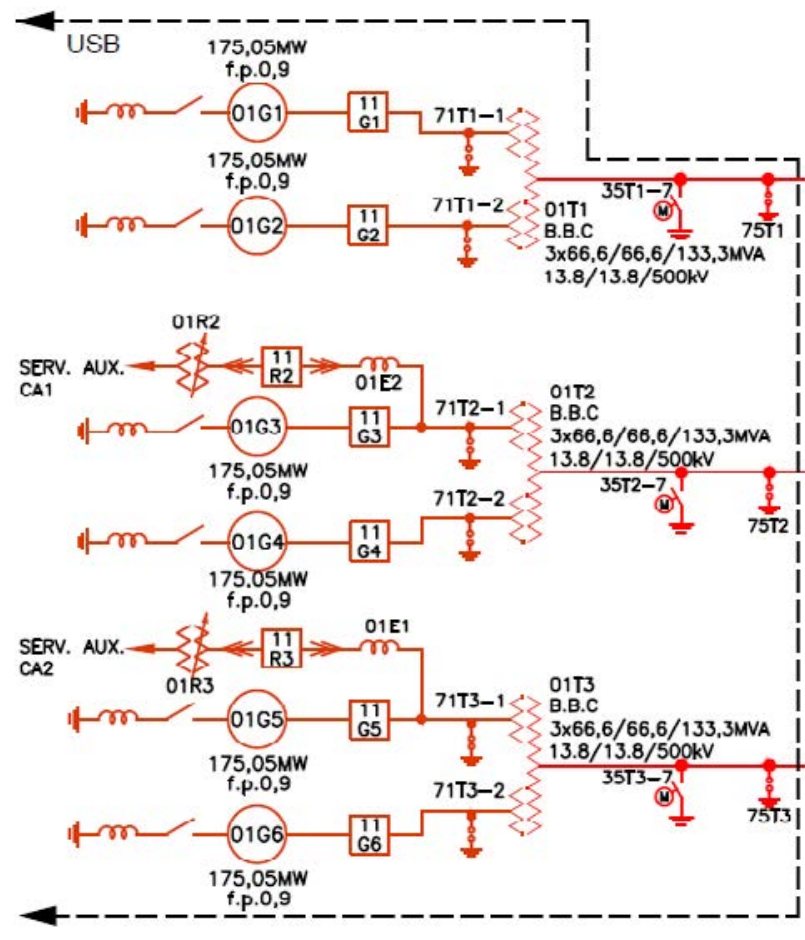

Fonte: Matos (2017).

Os geradores de emergência (GGE) são responsáveis pelo processo de partida dos geradores principais. Após a partida os serviços auxiliares da usina são alimentados através de duas fontes de serviços auxiliares 11R2/01R2 e 11R3/01R3, a partir daí dá-se prosseguimento ao processo de reenergização do sistema.

\section{Metodologia}

O objetivo deste estudo foi analisar o fluxo de carga durante a fase fluente do processo de reenergização do subsistema sudoeste. Para tanto, foi utilizado o programa PowerWorld Simulator. Esse programa utiliza métodos matemáticos (Newton-Raphson) para solucionar o fluxo de carga.

Para modelar o sistema no programa foi necessário obter os dados de placa de todos os equipamentos que fazem parte do sistema e os parâmetros das linhas de transmissão. Após isso, foram efetuados os cálculos de mudança de base em pu, para a base $\mathrm{Sb}=100 \mathrm{MVA} \mathrm{e} \mathrm{Vb}=500 \mathrm{kV}$, em conformidade com as diretrizes do ONS. Com as impedâncias na mesma base, os valores foram lançados no diagrama de impedâncias do PowerWorld, juntamente com os limites de operação dos equipamentos.

Nas Tabelas 1 e 2 estão especificados os limites de tensões e frequência durante todas as simulações que foram realizadas. Estes valores foram definidos em acordo com os agentes do setor (ONS, 2017f). O respeito a esses limites é essencial para evitar danos, colaborar no processo de regulação de tensão e para que as tensões cheguem em níveis adequados ao consumidor final.

Tabela 1 - Faixas de tensão

Tensão nominal do barramento (kV)

\section{0}

230

138

69
Faixa de tensão (kV)

450 a 550

207 a 242

124 a 145

62 a 72

Fonte: ONS (2017f). 
Tabela 2 - Faixa de frequências permitidas $(\mathrm{Hz})$

\section{Faixa de frequência $(\mathrm{Hz})$}

Fase fluente

58 e 62

\section{Fase coordenada}

59 e 61

Fonte: ONS (2017f).

A Figura 5 traz um exemplo da tela do programa PowerWorld durante uma das simulações realizadas. Nela é possível ver uma parte do subsistema representado com gráficos de setores indicando a porcentagem de carregamento de cada equipamento. Na Figura 5, as setas em cor verdes indicam o sentido do fluxo de potência ativa e, as setas em cor azul indicam o sentido do fluxo de potência reativa.

Figura 5: Programa PowerWorld Simulator.

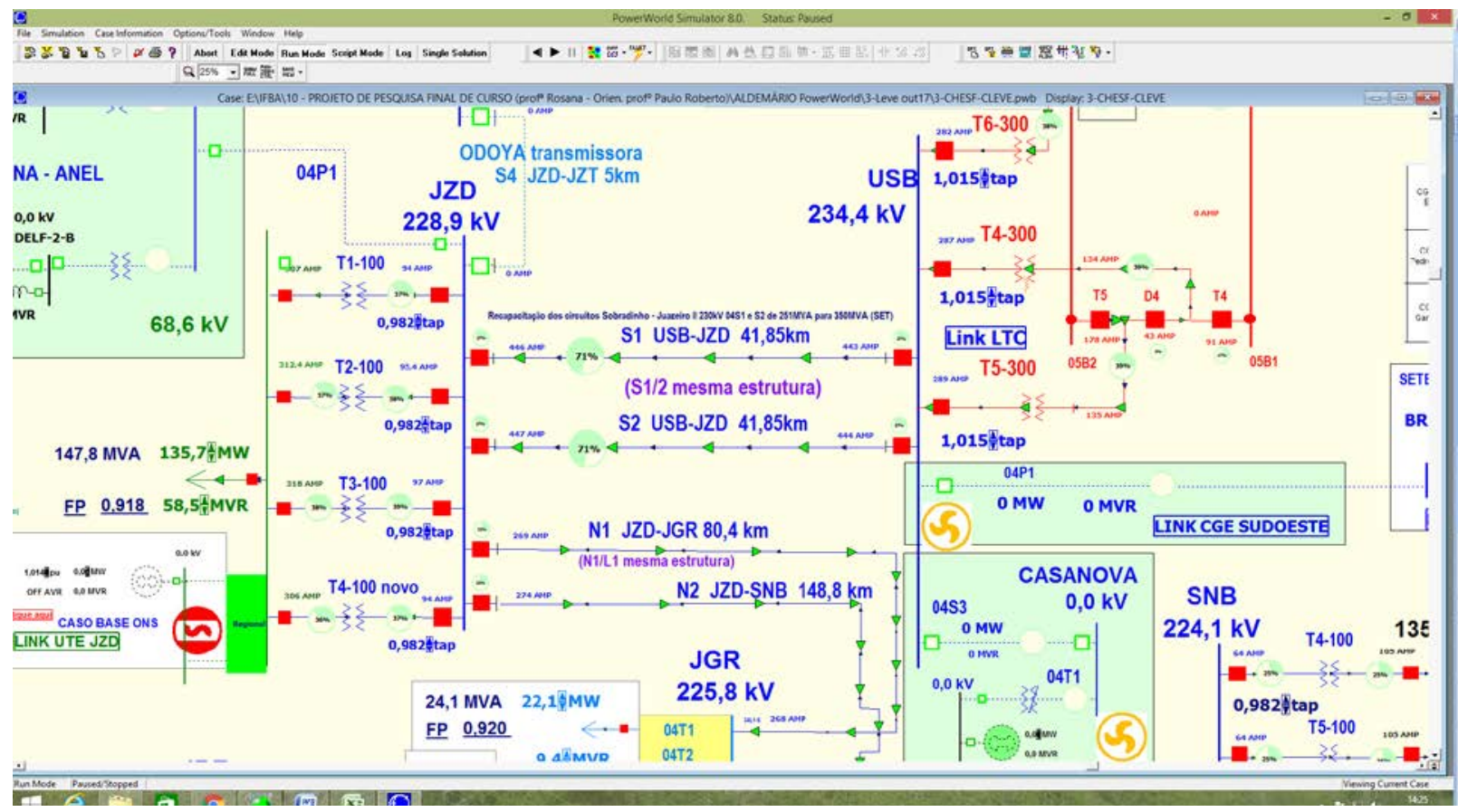

Fonte: Elaboração Própria (2017).

Para análise do fluxo de potência durante a reenergização do sistema, foi realizada simulação no Power World, conforme a instrução de operação do nos, que orienta o processo de recomposição da área SobradinhoSudoeste do Nordeste (ONS, 2017f).

\section{Resultados e discussão}

A sequência de reenergização do sudoeste da região Nordeste foi realizada como preconiza a instrução de operação do ONS para área (ONS, 2017f). A simulação no PowerWorld foi realizada com sucesso e não houve dificuldades na execução.

Em um primeiro momento, foram analisados os valores de tensão, em porcentagem dos valores nominais dos equipamentos da malha principal do subsistema. Os resultados estão indicados na Tabela 3 e no Gráfico 1. Neles é possível observar que todas as tensões ficaram dentro dos limites permitidos pelo ONS. Assim, em conformidade com o ONS (ONS, 2017f), a tensão de geração na usina de Sobradinho foi ajustada para 13,1 kV. 
Tabela 3 - Tensões da malha principal

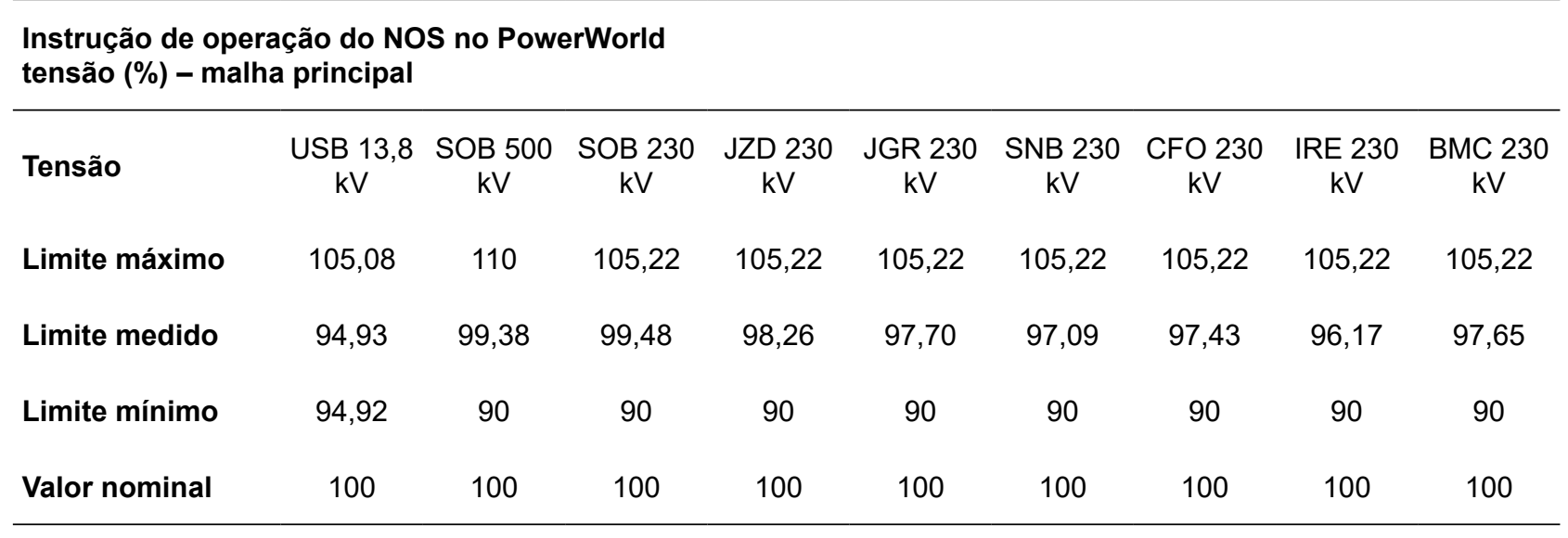

Fonte: Elaboração própria (2017).

Gráfico 1: Tensões na malha principal

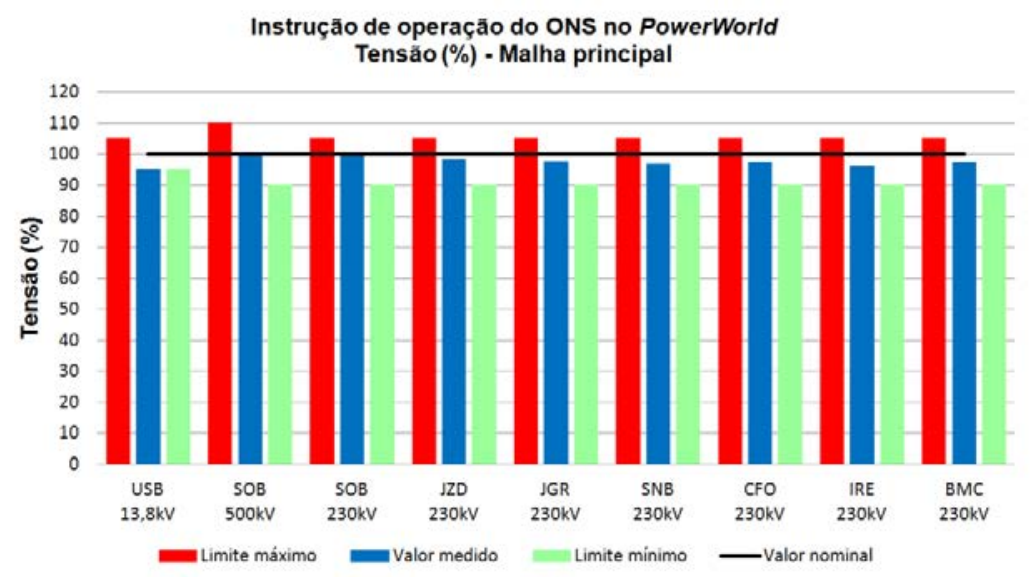

Fonte: Elaboração própria (2017).

Um ponto importante a destacar é que todos os equipamentos apresentam folga para variações de tensões, tanto para o limite inferior quanto para o limite superior, com exceção apenas da USB que se encontra, por força da instrução normativa, no limite inferior. Pode-se observar também que a subestação SOB opera próxima ao valor nominal, tanto no setor de $500 \mathrm{kV}$ como no setor de $230 \mathrm{kV}$.

Outro objeto de análise pertinente é a tensão nas barras de conexão com as distribuidoras. O quadro aqui é semelhante ao que se observa na malha principal. Os valores medidos na simulação encontram-se dentro dos limites estabelecidos pelo ONS. Verifica-se então que existe uma folga para atuação da regulação, o que permite um aumento controlado no montante de carga. Verificou-se que nenhum dos valores medidos ficou próximo ao valor nominal, garantindo uma faixa de segurança ainda maior. Os resultados para os valores de tensões na barra de carga podem ser vistos na Tabela 4 e no Gráfico 2.

Tabela 4 - Tensões na barra de carga

\begin{tabular}{lccccc}
\hline $\begin{array}{l}\text { Instrução de operação do ONS no PowerWorld } \\
\text { tensão (\%) - Na carga }\end{array}$ & JZD & SNB & SNB & IRE & IRE \\
\hline Tensão & $69 \mathrm{kV}$ & $138 \mathrm{kV}$ & $69 \mathrm{kV}$ & $138 \mathrm{kV}$ & $69 \mathrm{kV}$ \\
Limite máximo & 104,35 & 105,08 & 104,35 & 105,08 & 104,35 \\
Valor medido & 65,36 & 95,72 & 96,23 & 96,09 & 96,52 \\
Limite mínimo & 89,85 & 89,85 & 89,85 & 89,85 & 89,85 \\
Valor nominal & 100 & 100 & 100 & 100 & 100 \\
\hline
\end{tabular}

Fonte: Elaboração própria (2017). 
Gráfico 2: Tensões na barra de carga

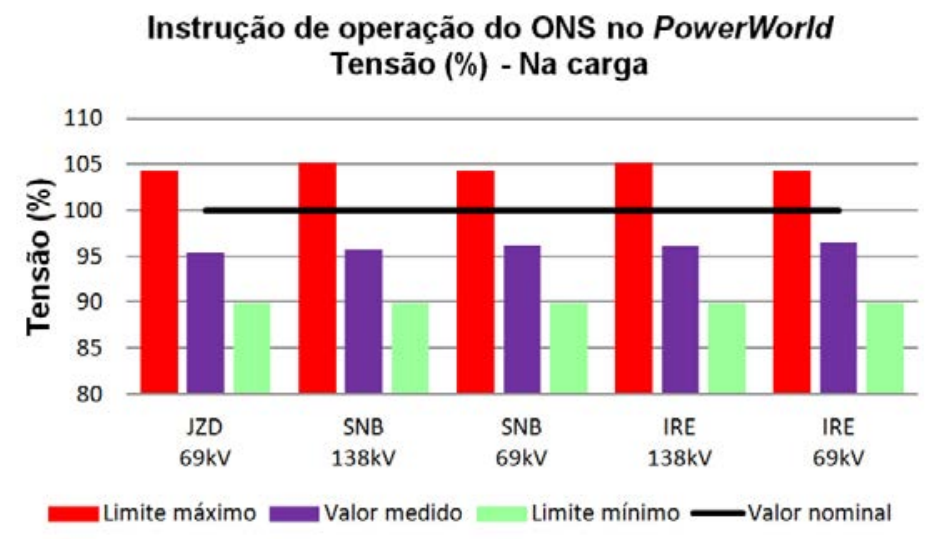

Fonte: Elaboração própria (2017)

Ainda pode-se analisar a evolução da carga durante o processo de reenergização. Após a conclusão dos procedimentos normativos, verificou-se que a porcentagem das cargas restabelecidas por subestação é inferior ao valor nominal da capacidade de transformação das mesmas, bem como ao máximo histórico registrado (CHESF, 2017). Os dados mostrados na Tabela 5 e no Gráfico 3 indicam que existe uma folga considerável para elevação de carga em cada setor. Os valores da máxima carga histórica estão disponíveis no Sistema Aberto de Gerenciamento de Energia da CHESF - Companhia Hidrelétrica do São Francisco (CHESF, 2017).

Tabela 5 - Carga por subestação

\begin{tabular}{lcccccc}
\hline $\begin{array}{l}\text { Instrução de operação do ONS no PowerWorld } \\
\text { carga por subestação (\%) }\end{array}$ & \multicolumn{1}{l}{ JZD } & JGR & SNB & SNB & IRE & IRE \\
\hline Carga & $69 \mathrm{kV}$ & $230 \mathrm{kV}$ & $138 \mathrm{kV}$ & $69 \mathrm{kV}$ & $138 \mathrm{kV}$ & $69 \mathrm{kV}$ \\
& 100 & 100 & 100 & 100 & 100 & 100 \\
Valor nominal & 75,60 & 22,23 & 79,28 & 33,15 & 63,48 & 100,16 \\
Máxima histórica & 25,50 & 13,62 & 16,50 & 16,00 & 28,00 & 31,67 \\
Valor medido (\%) do limite de transformação & & & &
\end{tabular}

Fonte: Elaboração própria (2017).

Gráfico 3: Carga por subestação.

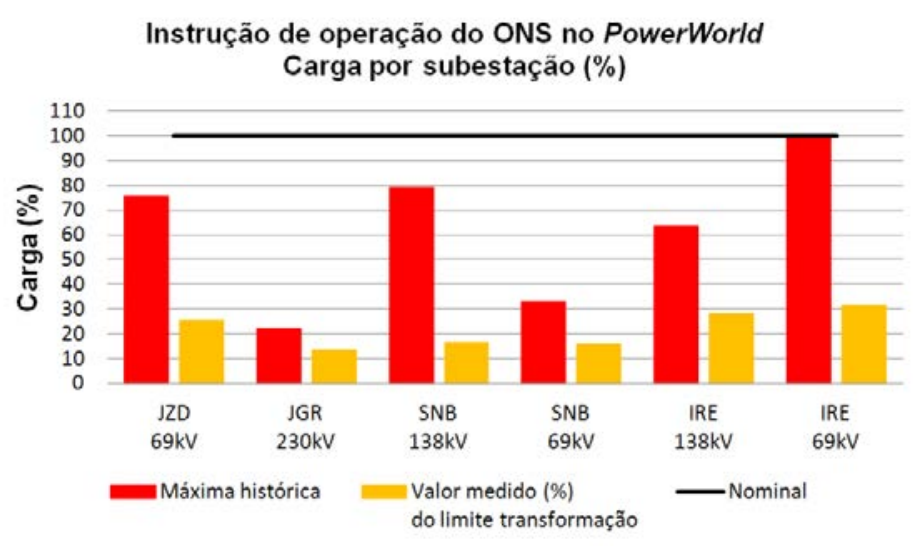

Fonte: Elaboração própria (2017). 
A Tabela 6 mostra os dados de carga dos transformadores que participaram da simulação. Por conveniência do ONS alguns transformadores, não participam da operação, a saber: 05T6 SOB, 04T2 JZD, 04T5 SNB, 04T3 IRE e 04T5 IRE (ONS, 2017f).

Tabela 6 - Carga por transformador

\begin{tabular}{|c|c|c|c|c|c|c|c|c|c|c|c|c|c|c|c|c|c|}
\hline \multicolumn{18}{|c|}{$\begin{array}{l}\text { Instrução de operação do ONS no PowerWorld } \\
\text { Carga por transformador (\%) }\end{array}$} \\
\hline Carga & $\begin{array}{l}\text { SOB } \\
\text { 05T4 }\end{array}$ & $\begin{array}{l}\text { SOB } \\
\text { 05T5 }\end{array}$ & $\begin{array}{l}\text { SOB } \\
\text { 05T6 }\end{array}$ & $\begin{array}{l}\text { JZD } \\
\text { 04T1 }\end{array}$ & $\begin{array}{l}\text { JZD } \\
04 T 2\end{array}$ & $\begin{array}{l}\text { JZD } \\
\text { 04T3 }\end{array}$ & $\begin{array}{l}\text { JZD } \\
\text { 04T4 }\end{array}$ & $\begin{array}{c}\text { JGR } \\
04 T 1 / T 2\end{array}$ & $\begin{array}{l}\text { SNB } \\
04 T 1\end{array}$ & $\begin{array}{l}\text { SNB } \\
04 T 3\end{array}$ & $\begin{array}{l}\text { SNB } \\
04 T 4\end{array}$ & $\begin{array}{l}\text { SNB } \\
04 T 5\end{array}$ & $\begin{array}{l}\mathrm{IRE} \\
04 \mathrm{~T} 1\end{array}$ & $\begin{array}{l}\text { IRE } \\
04 T 2\end{array}$ & $\begin{array}{l}\text { IRE } \\
04 T 3\end{array}$ & $\begin{array}{l}\text { IRE } \\
\text { 04T4 }\end{array}$ & $\begin{array}{l}\text { IRE } \\
\text { 04T5 }\end{array}$ \\
\hline Valor medido & 37 & 38 & 0 & 50 & 0 & 52 & 0 & 13,62 & 16 & 16 & 33 & 0 & 50 & 45 & 0 & 56 & 0 \\
\hline Nominal & 100 & 100 & 100 & 100 & 100 & 100 & 100 & 100 & 100 & 100 & 100 & 100 & 100 & 100 & 100 & 100 & 100 \\
\hline Máxima histórica & - & - & - & 77,69 & 74,90 & 80,88 & 68,92 & 22,23 & 33,15 & 33,15 & 79,28 & 79,28 & 124,49 & 91,00 & 85,00 & 63,48 & 63,48 \\
\hline
\end{tabular}

Fonte: Elaboração própria (2017).

Percebe-se que existe espaço para aumentar o carregamento sem risco de ultrapassar os limites nominais de operação, especialmente quando os demais transformadores forem energizados, o que significa um aumento na capacidade total de transformação.

Na Tabela 7 são exibidos os resultados da carga por linha de transmissão. Nela é possível ver que todas operam com baixo carregamento, o que era previsto pela instrução normativa em análise. A linha de transmissão LT 04F2 IRE/BMC apresenta 0\% de carregamento por ela ser uma subestação apenas de geração.

Tabela 7 - Carga por linha de transmissão

\begin{tabular}{lcccccccc}
\hline $\begin{array}{l}\text { Instrução de operação do ONS no PowerWorld } \\
\text { Carga por linha de transmissão }\end{array}$ & $\mathbf{2 3 0} \mathbf{~ k V ~ ( \% )}$ \\
\hline Carga & LT 040S1 & LT 04S2 & LT 04N1 & LT 04N2 & LT 04L1 & LT 04C1 & LT 04F1 & LT 04F2 \\
\hline VOB/JZD & SOB/JZD & JZD/JGR & JZD/SNB & JGR/SNB & SNB/CFO & CFO /IRE & IRE/BMC \\
Valor medido & 47,00 & 47,00 & 29,00 & 29,48 & 22,35 & 27,00 & 26,00 & 0 \\
Valor nominal & 100 & 100 & 100 & 100 & 100 & 100 & 100 & 100 \\
\hline
\end{tabular}

Fonte: Elaboração própria (2017).

\section{Conclusão}

A modelagem do sistema via o programa PowerWorld se mostrou satisfatória. As qualidades do simulador, especialmente o ótimo design gráfico e usabilidade facilitada, contribuíram para o êxito das simulações e a coleta de dados consolidados.

Verificou-se que os dados obtidos convergem para aqueles indicados pelo ONS na instrução normativa que foi a base para este trabalho. Todos os resultados ficaram dentro dos limites máximo e mínimo estabelecido pelo regulador do sistema, a fim de que o processo de reenergização seja operado com o máximo de segurança e qualidade.

Outra constatação relevante é que tanto os limites nominais da capacidade de carga das subestações, como das linhas de transmissão não foram alcançados, sendo o valor máximo obtido no caso das subestações de $31,67 \%$ na SE IRE, conforme pode ser observado na Tabela 5. É o mesmo no caso das linhas de transmissão de $47 \%$ nas linhas LT 04S1 e 04S2 entre as subestações SOB e JZD de acordo com a Tabela 7. Estes dados indicam a possibilidade de elevação de cargas no subsistema estudado.

Desta forma, pode-se considerar que o programa PowerWorld se mostrou uma importante ferramenta para análise de fluxo de cargas no sistema elétrico, apresentando dados em conformidade com os estudos e instruções do ONS, além de indicar as capacidades operacionais do sistema facilitando a compreensão do processo de reenergização e apontando para a otimização dos recursos de regulação. 
Propõe-se, então, como próximo passo deste estudo, a busca pela otimização dos recursos de regulação no processo de reenergização do subsistema analisado.

\section{Referências}

COMPANHIA HIDRO ELÉTRICA DO SÃO FRANCISCO (CHESF). IO.B.03 Excursionamento de LTC e tape fixo de trafos: Manual da operação MO: Instrução de operação IO. 35 ed. vigência: 19 set. 2017. Recife: CHESF, 2017.

KINDERMANN, Geraldo. Curto-circuito. 5.ed. Florianópolis: LabPlan, 2010.

MATOS, Aldemário Gomes de. Análise do fluxo de carga durante a reenergização do SIN: estudo de caso no trecho usina Sobradinho à subestação 230 kV Brotas de Macaúbas. 2017. Trabalho de Conclusão de Curso (Bacharelado em Engenharia Elétrica) - Coordenação de Engenharia Elétrica, IFBA, Paulo Afonso-BA, 2017.

OPERADOR NACIONAL DO SISTEMA ELÉTRICO (ONS). IO-RR.NE - Recomposição das interligações das áreas da região nordeste: rev. 13 - item 3.4.3 - 08 nov. 2016: Procedimentos de rede. Manual de Procedimentos da Operação. Módulo 10 - Submódulo 10.21. Brasília: ONS, 2017a. Disponível em: http:// ons.org.br/pt/paginas/sobre-o-ons/procedimentos-de-rede/mpo> Acesso em: 09 out. 2017.

OPERADOR NACIONAL DO SISTEMA ELÉTRICO (ONS). Plano da operação energética 2017/2021. Brasília: ONS, 2017b. Disponível em: http://www.ons.org.br/AcervoDigitalDocu-mentosEPublicacoes/RE-30108 2017_PEN2017_SumarioExecutivo.pdf. Acesso em: 04 out. 2017.

OPERADOR NACIONAL DO SISTEMA ELÉTRICO (ONS). Submódulo 20.1 Glossário de termos técnicos: rev. 2016.12 - 01 jan, 2017. Procedimentos de rede. Módulo 20 - Glossário de termos técnicos. Brasília: ONS, 2017c. Disponível em: http://www.ons.org.br/pt/paginas/sobre-o-ons/procedimentos-derede/ vigentes. Acesso em: 09 out. 2017.

OPERADOR NACIONAL DO SISTEMA ELÉTRICO (ONS). Submódulo 10.11 Recomposição da rede de operação após perturbação: rev. 2016.12 - 01 jan. 2017. Procedimentos de rede. Módulo 10. Brasília: ONS, 2017d. Disponível em: http://www.ons.org.br/pt/paginas/sobre-o-ons/procedimentos-derede/vigentes. Acesso em: 20 jul. 2017.

OPERADOR NACIONAL DO SISTEMA ELÉTRICO (ONS). RO-RR.BR.01 Testes reais de recomposição nas usinas de autorrestabelecimento: rev. 21 - item 4.1.6 - 15 out. 2017. Procedimentos de rede. Módulo 10 - Submódulo 10.22. Brasília: ONS, 2017e. Disponível em: http://ons.org.br/pt/paginas/sobre-o-ons/ procedimentos-de-rede/mpo. Acesso em: 09 out. 2017.

OPERADOR NACIONAL DO SISTEMA ELÉTRICO (ONS). IO-RR.NE.SBSO - Recomposição da área sobradinho - Sudoeste do Nordeste: rev. 6 - item 3.4.3 - 19 jun. 2017. Procedimentos de rede. Manual de Procedimentos da Operação. Módulo 10 - Submódulo 10.21. Brasília: ONS, 2017f. Disponível em: http:// ons.org.br/pt/paginas/sobre-o-ons/procedimentos-de-rede/mpo. Acesso em: 20 jul. 2017.

OPERADOR NACIONAL DO SISTEMA ELÉTRICO (ONS). CD-CT.NE.2SO.02 - Cadastro de limites operacionais de linhas de transmissão e transformadores da área $230 \mathrm{kV}$ oeste da região nordeste: rev. 26. item 2.2.3. 05 dez. 2016. Procedimentos de rede. Manual de Procedimentos da Operação. Módulo 10 - Submódulo 10.18. Brasília: ONS, 2017g. Disponível em: http://ons.org.br/pt/paginas/sobre-o-ons/ procedimentos-de-rede/mpo Acesso em: 09 out. 2017.

OPERADOR NACIONAL DO SISTEMA ELÉTRICO (ONS). Atuação: Mapas: Reservatórios. Brasília: ONS, 2020. Disponível em: http://www.ons.org.br/paginas/sobre-o-sin/mapas. Acesso em: 25 fev. 2020.

SOFTWARE SIMULADOR de fluxo de carga. Powerworld Corporation, Champaign-USA, [2017]. Disponível em: https://www.powerworld.com/. Acesso em: 23 nov. 2017. 
STEVENSON, Willian D. Elementos de análise de sistemas de potência. 2. ed. São Paulo: McGraw-Hill, 1986.

\section{Sobre os autores}

\section{Aldemário Gomes de Matos}

Engenheiro eletricista pelo Instituto Federal de Educação, Ciência e Tecnologia da Bahia (IFBA), Especialista em Educação à Distância pela Universidade do Estado da Bahia (UNEB) Campus I, licenciado em Ciências com habilitação em Matemática pela Universidade do Estado da Bahia (UNEB) Campus VIII, Técnico em Eletrotécnica pela Escola Técnica Federal de Pernambuco (ETFPE), Operador do Sistema Eletrotenergético na Companhia Hidro Elétrica do São Francisco - CHESF há 22 anos.

\section{Paulo Roberto Ribeiro Morais}

Graduado em Engenharia Elétrica pelo Instituto de Federal de Educação, Ciência e Tecnologia da Bahia (IFBA), Especialista em Sistemas de Telecomunicações pela Escola Superior Aberta do Brasil (ESAB), Professor do Ensino Básico, Técnico e Tecnológico do IFBA ligado à Coordenação de Eletrotécnica do Campus Feira de Santana.

Recebido em: 22.04 .2020

Aceito em: 20.09.2021 